\title{
DE LA CONCEPTUALIZACIÓN PEDAGÓGICA A LA CONSTRUCCIÓN DE UNA PEDAGOGÍA CRÍTICA PARA LA ATENCIÓN A LA DIVERSIDAD
}

El presente artículo, se justifica en la medida que incorpora las preocupaciones temáticas básicas que nuestro nuevo diseño curricular provoca en cuanto al tratamiento de la diversidad desde la pedagogía crítica en los planes de Formación de Formadores. Constituye, además, una problemática de primer orden y la orientación fundamental del Plan de estudios del posgrado en Pedagogía con Énfasis en Diversidad en los Procesos Educativos que se ofrece en la División de Educación Básica del Centro de Investigación y Docencia en Educación de la Universidad Nacional.

\author{
Paulette Barberoussel \\ Luz Emilia Flores Davis ${ }^{2}$ \\ Rafael Estéban Jiménez Corrales ${ }^{3}$
Irma Zúñiga León \\ Rafael Estéban Jiménez Corrales
Irma Zúñiga León
}

This paper presents and justifies the basic concerns aroused by our new curriculum design; concerns that are related with the treatment given to diversity issues from the perspective of critical pedagogy in teacher training programs. We address here an important problem and issue that constitutes the basic orientation of the Graduate Program on Pedagogy with an Emphasis on Diversity in Educational Processes, which is offered by the Division on Elementary Education of the Universidad Nacional's School of Education (Spanish acronym, CIDE).

1 Máster en Ciencias Históricas y Filosóficas de la Universidad de Babes - Bolyai de la República de Rumania. Máster en Ciencias de la Educación con énfasis en Educación de Adultos de la Universidad de Costa Rica. Académica División de Educación Básica, CIDE-UNA.

2 Máster en Educación Superior por la Universidad Regiomontana de Monterrey, México. Académica de la División de Educación Básica, CIDE-UNA.

3 Bachiller y Licenciado en Historia de la Universidad de Costa Rica. Estudios en la Enseñanza de los Estudios Sociales de la Universidad de Costa Rica. Máster en Evaluación Educativa de la Universidad de Costa Rica. Subdirector División de Educación Básica, CIDE-UNA.

4 Bachiller en Ciencias de la Educación con Especialidad en Preescolar y Administración Educativa de la Universidad de Costa Rica. Licenciada en Ciencias de la Educación con especialidad en la Enseñanza Preescolar de la Universidad Nacional. Bachiller en Pedagogía de la Comunicación de la Universidad Nacional. Máster en Desarrollo del niño y relaciones familiares de la Universidad de Carolina del Este, Estados Unidos de América. Actual decana del CIDE - UNA. 
Dado el hecho de que el tratamiento pedagógico de la diversidad se inscribe como temática emergente en el contexto de la educación superior, un grupo de colegas universitarios nos dimos a la tarea de reunimos y extemar una serie de reflexiones y comentarios que tuvieron como hilo conductor la idea de cómo se ha abordado la diversidad desde el enfoque crítico, tratando de relacionarlo con la diferencia que se establece entre la perspectiva desde las Ciencias de la Educación o de la Pedagogía, tomando en consideración las actuales reelaboraciones de la conceptualización misma de la pedagogía crítica, los enfoques y tendencias dentro del tratamiento de la diversidad multicultural así como las interrelaciones entre sociedad-desigualdad-diversidad.

\section{La diversidad en el marco de la Pedagogía}

El tema de la diversidad en el ámbito de la educación puede ser abordado desde una amplia gama de propósitos y perspectivas, por lo que un aspecto por definir es si el estudio se realizará desde el marco conceptual de las Ciencias de la Educación o de la Pedagogía.

Cabe señalar que la falta de definición en tomo a la polémica: ¿Ciencias de la Educación o Pedagogía? ha generado de por sí, confusiones que obstaculizan el desarrollo de ambas, así como el uso de uno u otro concepto de manera indistinta. Un ejemplo de ello es la acreditación de profesionales en el campo de la educación otorgando títulos en Ciencias de la Educación, cuando la formación corresponde más bien a la Pedagogía, o al contrario, cuando encontramos profesionales titulados en Pedagogía con el respaldo de un plan de estudios que más bien corresponde a las Ciencias de la Educación.

En razón de lo anterior es conveniente tener presente que tanto las Ciencias de la Educación como la Pedagogia, estudian la educación con propósitos distintos y ocupándose de aspectos diferentes.

¿A qué se refieren las Ciencias de la Educación?

Las Ciencias de la Educación tienen por objeto de estudio, los hechos o fenómenos educacionales, generalmente con un interés predominantemente descriptivo. Abarca varias disciplinas con el propósito de analizar distintos aspectos de la educación. Entre ellas se encuentran la Psicología de la Educación, la Sociología de la Educación, la Economía de la Educación, la Historia de la Educación, entre otras.

Como puede observarse, cada una de las disciplinas que conforman las Ciencias de la Educación tienen su propia ciencia de origen, a saber la Psicología, la Sociología, la Antropología. Además, cada una de estas disciplinas es desarrollada por sus propios especialistas, por ejemplo, quien se ocupa de la 
La Pedagogía

conceptualizada desde hace algunos años como crítica, y entendida como aquella o aquellas propuestas pedagógicas que realizan planteamientos contestatarios frente a la Pedagogía o Pedagogias oficiales $y$ sostenidas por las estructuras políticas oficiales no constituyen esencialmente formas del todo inéditas.
Sociología de la Educación es el sociologo, ciertamente con interés por la educación, pero desde su campo de especialidad. Por lo tanto, no existe un especialista para todas las ciencias de la educación, dado que cada una conserva su identidad y su relacion con la ciencia que le da origen y cuyo objeto de estudio no se circunscribe únicamente al ámbito de la educación.

Inicialmente estas disciplinas fueron consideradas como auxiliares de la Pedagogía, sin embargo, su de. sarrollo ha sido tal que han dado origen a la categoría de Ciencias de la Educación.

El desarrollo de cada una de las ciencias de la educación si bien constituye un aporte significativo en el avance de la educación, requiere de un esfuerzoespecial del pedagogo para aplicar esos hallazgos a la realidad del aula escolar. La Psicología de la Educación, por ejemplo, ha generado conocimiento relevante para comprender cómo se aprende, cómo intluyen las relaciones interpersonales en el aprendizaje y muchos otros aportes importantes para el trabajo en el aula; pero el aula constituye un espacio ( físico, social, cultural) que rebasa el campo de la psicología.

Y por otra parte: ¿Qué es la Pedagogía?

La Pedagogía, o si se quiere la Ciencia Pedagógica es la Ciencia de la Educación. "Ciencia" en singular. Tiene por objeto de estudio el acto educativo, la acción de educar, acción que implica práctica y teoría de manera integrada.

La Pedagogía toma en consideración los aportes de las Ciencias de la Esucación, pero no se diluye en ellas. Su campo de accion es el hecho educativo, por lo cual su desarrollo es responsabilidad del pedagogo o educador.

Así como complejo es el hecho educativo, así también es compleja la Ciencia Pedagógica, la cual adquiere la categoria de ciencia por tencr un objeto de estudio y un método propio, lo cual no impide que se reconozca que la pedagogía, además de ciencia tiene mucho de arte, pues cada praxis educativa es única, original c irrepetible y requiere ser creada por los protagonistas de cada hecho pedag6gico.

La Pedagogía se ocupa de la praxis educativa en sus concrctizaciones institucionalizadas y no institucionalizadas correspondiente a la atención de niños, jóvenes y adultos, tanto en el nivel individual como en el colectivo. Está conformada por 
diversas disciplinas tales como la didáctica, el currículum, la evaluación de la educación, la gestión educativa.

A través de los años la Pedagogía ha evolucionado, en la búsqueda de altemativas de solución a los problemas encontrados, así como al avance de las Ciencias de la Educación y a las concepciones de realidad y a los fines y metas de formación que se pretenden alcanzar por medio de la educación. Ello ha generado tendencias tales como la Pedagogía Tradicional, la Conductista, la Pedagogía Romántica, la Pedagogía Crítica, y la Cognitiva, entre otras.

Entonces : ¿Ciencias de la Educación o Pedagogía?

Una vez delimitado el objeto de estudio de las Ciencias de la Educación y la Pedagogía y teniendo claridad de los alcances de cada una de ellas, podemos valorar la importancia para la educación del desarrollo de ambas. De esta manera podemos ubicar el estudio del tema de la diversidad en el marco de las Ciencias de la Educación o de la Pedagogía, según los propósitos que se persigan.

Clarificados los ámbitos conceptuales tanto de la Pedagogía como de las Ciencias de la Educación, nos abocamos a la clarificación del cuestinoamiento en tomo a la posibilidad de existencia de una Pedagogía denominada crítica, es decir, una ciencia de la educación que además del análisis del objeto de estudio "educación", le dé un nivel precscriptivo hacia principios de criticidad. No obstante, para visualizar hacia dónde se dirige esa criticidad se analizarán algunas de las corrientes pedagogicas que historicamente se han hecho acreedoras del apelativo de críticas.

\section{La criticidad de la Pedagogía a través de la historia}

¿Criticidad en Pedagogía: novedad o constante histórica?, he ahí una de las principales interrogantes que se deben formular en la clarificación históricoconceptual de la hoy llamada Pedagogía Crítica.

La Pedagogía conceptualizada desde hace algunos años como crítica, y entendida como aquella o aquellas propuestas pedagogicas que realizan planteamientos contestatarios frente a la Pedagogía o Pedagogías oficiales y sostenidas por las estructuras políticas oficiales no constituyen esencialmente formas del todo inéditas.

Desde principios de siglo $\mathrm{XX}$ difcrentes propuestas han constituido formas alternativas de entender y ejecutar los procesos pedag6gicos. Desde los enfoques románticos, hasta las críticas marxista estructuralistas, pasando por las propuestas de la escuela activa, la desescolarizacion, y muchas otras, han cuestionado elementos tradicionales de los sistemas educativos tales como la rigidez, autoritarismo, y coerción, pero no todos ellos han buscado enlaces 
directos entre las microtendencias escolares de aula y las macrotendencias sociales, económicas y políticas, es decir no llevan explicitado un cuestionamiento sistemático de la función social que la práctica de aula produce en el entramado social mayor.

Uno de los aportes fundamentales a las posiciones pedagógicas críticas proviene del pensamiento marxista, pero ante todo de la lectura de tal propuesta, hecha por Antonio Gramsci. El cual a pesar de no tener como objeto de estudio "per se" a la Pedagogía, desarrolla una importante teorización en torno al papel y conformación del Estado dentro de una sociedad capitalista. Gramsci a partir del concepto totalizador de Bloque Histórico, sienta las bases de la inteıpretación de un Estado de clase sustentado en formas ideológicas y coercitivas de dominación. El autor italiano ubica al fenómeno educativo en general, como un mecanismo ideológico básico de generar y mantener procesos de dominación y reproducción social.

Las ideas gramscianas son retomadas por diferentes autores dentro de los cuales destaca Louis Althouser y sus múltiples seguidores. Esta corriente de análisis social propone el Modelo de la Teoría de la Reproducción Social. El cual sostiene que existe una correspondencia directa entre modelo educativo y sistema sociocconómico, o más estrictamente Modelo Societal (Rama, 1984). Es importante indicar que la propuesta de la Teoría de la Reproducción Social, nace al tomar como referente histórico la sociedad capitalista, de ahí que sus consideraciones se vean dirigidas a esa formación social en particular.

Según el Modelo de la Teoría de la Reproducción Social, la Educación, la escuela y la pedagogía son referentes y sustentadores directos de los modelos capitalistas de producción. Los sistemas educativos generan y transmiten ideas, formas organizativas, contenidos, mecanismos de evaluación, etc. Estos sosticnen y perpetúan un tipo de sociedad injusta y desigual: la sociedad capitalista.

Estas tendencias reproduccionistas reciben aportes de varios autores, pero ante todo una serie de críticas sustentadas en el carácter estructuralista en que se basan. Tal estructuralismo es parte consustancial de la tcoría marxista y su interpretación leninista. La misma, tiende a obviar las manifestaciones de los sujetos subalternos en los modelos capitalistas, manifestaciones como las formas contraculturales, procesos de resistencia desigual claborados por los sujetos involucrados dentro de las macroestructuras sociales.

Los críticos de los reproduccionistas consideran que en la larga instancia, la interpretación social y educativa realizada conlleva al inmovilismo, a la parálisis social, y quizá a una posible aceptación de los mecanismos establecidos. Sobre todo, al partir de la premisa de que el cambio social requiere un cambio estructural radical, sobre todo en la base económica. 
Una de las corrientes de pensamiento fundamentales que alimentan las concepciones pedagógicas críticas más recientes, lo es la emanada en la Escuela de Frankfurt. Dicha escuela de pensamiento ha dedicado sus esfuerzos teóricos a la crítica de la sociedad industrial y posindustrial, sus concepciones ideologicas y su funcionamiento social.

Las propuestas críticas emanadas desde la Escuela de Frankfurt proponen un cambio societal, en doble vía: individual y colectivo, que supere todo tipo de desigualdad, autoritarismo y alineación. (Ayuste, y otros, 1998). Desarrollan los principios de la denominada Teoría de la Acción Comunicativa (Habermas), concepción que constituye un replanteamiento ante las posiciones pedagógicas críticas estructuralistas, confiriendo acciones de cambio y modificación a los sujetos participantes en los procesos sociales. De la confluencia de la teoría reproduccionista y de las ideas de la Escuela de Frankfurt emergerá la Pedagogía Crítica norteamericana.

Por otra parte, dentro de las posiciones críticas en Pedagogía, se encuentra lo que algunos autores consideran como la Tendencia Latinoamericana, siendo su principal representante Paulo Freire. La obra freireana transita por diversas etapas o momentos, sin embargo, varios principios claves representan invariantes conceptuales tales como: el diálogo como elemento de concientización y transformación de la situacionalidad social de los sujetos, la confrontación entre pedagogías exclusionistas y pedagogias liberadoras como elementos de reproducción social, y la vinculación entre pedagogía y modelos societales.

A manera de sintesis se considera que la llamada Pedagogía Crítica constituye una propuesta educativa cuyo fines básicos lo son el cambio, la transformación social, económica y política. Actualmente persigue que las respuestas para los cambios y transformaciones surjan mediante procesos de diálogo y análisis crítico de la situacionalidad de los actores involucrados.

A la pregunta inicial ¿Criticidad en Pedagogia: novedad o constante histórica?, se puede responder de diversas maneras. La criticidad como elemento pedagógico se inicia desde el momento mismo en que la Pedagogía implica reflexión e inquietudes sobre el hecho educativo, pero la criticidad pedagógica como forma de articular pedagogía y cambio social, como elemento de denuncia y acción ante la exclusión, la marginalidad, y la injusticia social y educativa, tendria caminos o génesis posteriores. La Pedagogía Crítica como un corpus estructurado con principios integradores, y acciones didácticas concretas constituye una construcción conceptual relativamente reciente, y ante todo en permanente y constante conformación. Negar ese dinamismo, ese constante hacerse, ese carácter dialéctico y dialógico, sería negar la naturaleza misma de la Pedagogía Crítica. 


\section{La visibilización de la multiculturalidad como escenario de cambio en la Pedagogía Crítica}

Como se ha discutido anteriormente, gran parte de los modelos pedagógicos considerados críticos apuntaron hacia el cuestionamiento pedagógico de los sistemas educativos que generan exclusión. No obstante, en los movimientos iniciales de la Pedagogía Crítica, se da un marcado énfasis en la exclusión por clase social (herencia que no es de extrañar dadas las vinculaciones de la Pedagogía Crítica con posiciones marxistas y neomarxistas).

El dinamismo histórico de la sociedad, así como los cambios en los radios de análisis de las ciencias sociales, visibilizan otras formas de exclusión antes no contempladas a profundidad, donde destaca la exclusión por género y la exclusión por pertenencia cultural o étnica. En los siguientes párrafos se hará referencia a la relación entre Pedagogía y la visibilización de los procesos multiculturales.

La educación intercultural asumida como la convivencia de diferentes grupos culturales en un mismo contexto (Sales y García, 1997, p. 79) es uno de los retos del sistema educativo que más controversias ha generado en los últimos tiempos y de las cuales nuestro país noestá ajeno. Como problemática multifactorial, ha sido estudiada por diversas disciplinas tales como la: Antropología, Psicología, Linglística y Pedagogía. También, desde esta perspectiva se relaciona con la: educación moral, democrática, global, para el desarrollo y la paz y ha generado diversas políticas, modelos educativos, programas y experiencias.

Investigaciones de Torres, Perienoud, la Corte Suprema de Justicia de Costa Rica y Leach (citados por Alvarado y Rodríguez, 2000) ponen en evidencia actitudes de hostilidad y prejuicios ante la presencia de una persona distinta y el papel fundamental de la educación ya sea para imponer la cultura dominante, preparar a los docentes para manejar adecuadamente sus estereotipos frente a niños "visibles" o "invisibles", "bonitos", "simpáticos", "inteligentcs", "fcos" y "conflictivos" o respetar la diversidad étnica y cultural.

Como fenómeno social y político se origina en las reivindicaciones sociopolíticas de los 60's, desarrolladas en los Estados Unidos por grupos de negros, mujeres, homosexuales, discapacitados y pcrsonas de la tercera edad, cuyas presiones sentaron las bases para nuevos programas educativos. Asimismo, surge por el auge del factor étnico, como presión política frente a la disyuntiva de un Estado-nación y la necesidad del reconocimiento de los rasgos culturales y de la identidad propia.

Por otra parte, el fenómeno migratorio, del cual nuestro país es parte, hace evidente la influencia de personas que huyendo de problemas económicos, sociales y políticos llegan a Costa Rica buscando una mejor calidad de vida y nuevas oportunidades, dentro de las cuales se incluye la educación (Sandoval, 2000). Finalmente, 
según Sales y García (1997) la multiculturalidad se origina en la interdependencia mundial, la globalización y la existencia de onganismos y pactos internacionales que respaldan la defensa de los derechos de los diferentes grupos sociales y culturales.

La multiculturalidad se resume en la lucha por la igualdad de oportunidades ante la cual no hay neutralidad y ha sido asumida por las siguientes corrientes ideológicas y políticas.

\section{Asimilacionismo}

Surge en el siglo pasado y durante la I Guerra Mundial; aunque su preponderancia se da en los años 60 . Pretende absorber los grupos étnicos y culturales en una sociedad homogénea, porque la cultura dominante es superior desde la perspectiva política-económica y biológica. La adaptación debe darse también por razones económicas y de seguridad, porque la etnicidad es propia de las sociedades en vías de desarrollo, es una disfunción en virtud de que las sociedades avanzadas marchan hacia el universalismo. El asimilacionismo, así conceptualizado ha cedido a planteamientos más tolerantes.

La sociedad costarricense no escapa a la discriminación racial y su historia muestra como desde el siglo XIX se prohibe abiertamente la inmigración de razas africanas y chinas al país, declarándose indeseable a toda persona negra, china, siria o gitana y aunque desde 1948 se inicia un proceso de derogación de leyes discriminatorias, en la actualidad los costarricenses continúan mostrándose inconformes con los movimientos migratorios (Alvarado y otros, 2000).

\section{Integracionismo}

Es un eclectesismo étnico, que promueve la igualdad y unidad por medio de la diversidad. Busca evitar el conflicto y el desequilibrio social para mantener el orden establecido y los privilegios sociales, cediendo solamente en lo superficial. Se trata de amalgamar o crear una cultura común, que recoge el aporte de los diversos grupos, sin que ninguno se imponga; manteniendo la coexistencia y el equilibrio entre las culturas minoritarias y ofreciendo lo mejor de la cultura dominante para todos.

Es conocido en Estados Unidos como el metling pot o "crisol" y en Gran Bretaña, ha dado origen a la idea de un curriculum central para todos los educandos (core curriculum), acompañado de diversificación.

Se encuentra en un posición ambigua entre la igualdad de oportunidades y la teoría de la deficiencia, porque mantiene un sutil racismo y la superioridad de la cultura receptora. 
El fenómeno cultural es un problema de déficit cultural y la igualdad de oporıunidades es una homogenización de los modelos culturales lo que implicó poco respeto por la diversidad.

\section{Pluralismo}

Rechaza la diferencia cultural como positiva. Pretende que cada grupo cultural conserve y desarrolle su cultura en igualdad de condiciones. Se le suele denominar como salad bowl o fruit salad porque las distintas culturas, a partir de su autonomia, enriquecen la sociedad global. Busca que los individuos se comprometan con su grupo étnico, el cual ofrece identidad y apoyo psicológico, en la lucha por la reforma política y social.

A diferencia, del asimilacionismo, no considera que las culturas minoritarias sean deficitarias; sino que todas tienen el mismo estatus y el derecho a desarrollarse a plenitud por medio de espacios e instituciones propias. Cada grupo debe defender sus propios derechos.

Cae en relativismo cultural, que puede seguir discriminando los grupos minoritarios porque los aísla en su propia cultura. Asume la cultura como algo estático que no cambia con el tiempo y no considera la heterogeneidad intema de los grupos, por lo que niega el enriquecimiento producido por el intercambio intercultural.

\section{Interculturalismo}

Es más reciente y asume un concepto de cultura más dinámico y cambiante. Desde esta posición, ninguna cultura es superior a otra ni tiene derecho a dominarla; sin embargo, tampoco son iguales. La diversidad cultural es positiva y enfatiza en los elementos que pueden unir.

Pretende una reflexión continua y crítica de la propia cultura para destcrar aquello que contradice los valores universales. La sociedad es abierta y toclos pueden participar en igualdad de condiciones, por tanto, parte de supuestos de igualdad y justicia social para desarrollar relaciones sociales y políticas más justas y solidarias.

Úbica el factor étnico y cultural en su justa dimensión y lo interrelaciona con factores económicos, políticos, de género, clase social y de edad.

Desde la perspectiva educativa, defiende la igualdad de oportunidades para todos los alumnos, en términos de género, clase social, raza o cultura sentando las bases para la búsqueda de objetivos de igualdad, libertad y justicia, con miras a una sociedad justa, armónica, multirracial y multicultural. Olvida la lucha política, busca preservar los elementos culturales y lograr la igualdad educativa y social de todos los subgrupos mediante la igualdad en la 
estructura social. Por lo anterior, se busca eliminar las desigualdades por sexo, raza o cultura que pudieran justificar una distribución también desigual de los recursos y bienes sociales, para formar individuos interculturales y relaciones políticas justas y solidarias.

En síntesis, la educación multiétnica o multirracial se sustenta en los prejuicios raciales que se deben a la desigualdad económica y política y su evolución da origen a la educación multicultural. Esta olvida la lucha política y busca preservar elementos culturales, explicando las desigualdades por difcrencias culturales, se ha dirigido a grupos étnicos, de géncro, clase social y personas discapacitadas. Es la posición de países como Reino Unido, Estados Unidos, Australia y Canadá. La educación intercultural es sostenida en países de la Europa continental como Francia, Suiza y se ocupa de grupos étnicos y culturales de inmigrantes, pero además de toda la sociedad; aún de una comunidad relativamentc homogénea o monocultural. Esta propicia la interacción entre culturas, cn un ambiente de derecho a la diversidad y de igualdad de oportunidades, la flexibilización de los modelos culturales trasmitidos en la escuela, mayor rique$\mathrm{za}$, conocimiento y crítica reflexiva de los valores culturales propios y ajenos.

Cualquier Pedagogía que se considere a sí misma como crítica, debe tener como principio fundamental una perspectiva intercultural en el sentido que se ha desarrollado en líneas anteriores. Ese reto lleva necesariamente a plantearse una serie de interrogantcs en torno a la función de la Pedagogía Crítica o no en los escenarios actuales, lo cual se aborda seguidamente.

\section{Un diálogo abierto entre la Pedagogía Crítica y el reconocimiento de las diversidades}

Partamos de la basc que la misma divcrsidad que se presenta en la socicdad se reproducc en la escuela. Por esta razón, en los últimos años, el tema de la diversidad sc ha adueñado del debate pedagógico gencrando posturas disímiles en su comprensión y formas de actuar, no pocas veces antagónicas aún cuando blanden las mismas consignas. La misma desigualdad que existe en la sociedad se refleja, no mecánicamentc sino guardando las especificidades del contexto pedagógico, en la escuela. El problema es que en la socicdad la diversidad es considcrada como desigualdad y cn la cscuela como diversidad. Igualdad se opone a desigualdad, no a diferencia y diversidad. No se deben "pcdagogizar" los problemas sociales ya que la diversidad disfraza, enmascara y profundiza las desigualdades. La diversidad es lo normal, lo habitual, es lo positivo y enriquecedor. Es consustancial al género humano y un objetivo socialmente deseable. Ello exige creatividad, coherencia, compromiso, implicación y csperanza. Sin 
embargo, se ha considerado nornal lo que no es: la desigualdad que domina en la vida, en la sociedad y en la escuela. La escuela pública puede actuar como equilibradora de los desequilibrios sociales provocados por la desigualdad y la marginación, es decir, trabajar la diversidad para superar la desigualdad, entendiendo que la igualdad, soporte ético de la pedagogía de la diversidad, no significa uniformidad y bajo el mandato de compensar las desigualdades.

Al considerar a la escuela (en sentido genérico de institución escolar) reproductora del sistema social se evade la posibilidad de transformarla en agente de cambio con relativa autonomía y especificidad. Se debe de plano rechazar las posturas academicistas que tratan de "adaptarse" al statu quo, al igual que las posturas psicologizantes y didactistas que pretenden superar el fracaso escolar mediante el maquillaje de cambios exclusivamente metodológicos y que lo hacen recaer en el individuo cuando, en realidad, cumplen la misión de legitimar desigualdades sociales. Se debe reconstruir y repensar la escueladesde la diversidad para la igualdad.

En la praxis pedagógica lo diverso es lo habitual, lo excepcional es lo uniforme. Se toma necesario abordar el problema de la diversidad como un problema de desigualdad ante un currículo escolar que no es neutral ya que se asienta en su carácter y contenido ideológico. Esta ausencia de neutralidad le confiere a esta problemática una dimensión ideológica que afecta tanto las decisiones macropolíticas como a la intervención docente, guiada esta última por valores y creencias que conciben la homogeneidad como el paladín de la calidad cducativa cuando, en realidad, la heterogeneidad, enmarcada en un currículo comprensivo, abierto y flexible, único y diverso que se adapte al alumno y no a la inversa, constituye la única garantía de una educación fornativa y vivencial.

La atención a la diversidad, entendida desde la pedagogía crítica como lucha por la igualdad y como lucha por la construcción de una educación integradora y compensadora de desigualdades, implica elaborar proyectos educativos que contribuyan a compensar y superar las desigualdades en lugar de reproducirlas o aumentarlas; construir comunidades de aprendizaje cooperativo y reflexivo donde el diálogo se erija como principal mecanismo de construcción de significados interactivos e intersubjetivos del conocimiento. Para cumplir con tal asignación son necesarios equipos de profesores comprometidos con la educación pública que sean capaces de revolucionar su pensamiento y, sobre todo, su sensibilidad pedagógica como herramientas básicas de los procesos de crecimiento, de hominización y humanización a fin de lograr la tan ansiada realización humana. Es indispensable que conjuguen sus esfuerzos con las familias, los agentes y organizaciones de la sociedad civil, con el contexto real y afectivo de todos los integrantes de la comunidad educativa en pro de una educación para vivir con dignidad. 
Se trata de favorecer la igualdad de oportunidades y la comprensión de las desigualdades mediante una cnscñanza comprensiva $\mathrm{c}$ inclusiva como garante de la atención a la diversidad en sus más variadas modalidades y cxpresiones: cultural, étnica, de género, cognitiva, social, económica, política, religiosa, de acceso a información, de lugar que se ocupa en el proceso productivo, ética, moral, de sensibilidad, afectiva y emocional. Se siente distinto, se necesita distinto, actuamos distinto, pero se puede estimar y querer tal como son los diferentes grupos humanos si se logra establecer cauces de participación e interconexión entre las más variadas maneras de vivir, ccicbrar o mirar la vida.

Si la orientación y esfuerzos durante la década de los ochenta del debatc pedagógico sc centró en la búsqueda de una educación equitativa e igualitaria para todos y todas a través del aprendizaje significativo, la tendencia de fines del siglo pasado e inicios del presente ponc acento en la adaptación a la diferencia y la diversidad para lograr la transformación del contexto mediante cl aprendizaje dialógico. Deberían conformarse agrupamicntos flexibles que permiticran cl nacimicnto de una escuela plural que reconstruya críticamente la rcalidad y nuestra propia identidad desde una perspectiva liberadora con sentido personal y social y que formc para la participación democrática y ciudadana. No sc puede, sin embargo, dejar de exicmar la preocupación que el discurso sobre la atención a la diversidad se transformc hoy en un discurso que inmovilice, en la práctica, la potencialidad transformadora de la cscucla al adormecer su dimensión liberadora, justificando su status quo dotándolo de una perspectiva explicativa y científica de las desigualdades sociales y culturales. Y lo que se suponc más peligroso aún: creer y convencer que se puede solucionar desde y exclusivamente el ámbito pedagógico.

Si se cree que la cducación debc formar scres pensantcs, sc debe comenzar por pensar y pensar juntos. Castoriadis tienc razón cuando afirma que "nunca la acción educadora ha tenido más fuerza política que en la sociedad del "no-pensamiento". Es decir, como scres conscientcs, pueden desaprenderse a pensar como sc fue enseñado. Obviamente, cuando todo sc oponc a ello, es una tarea harto ardua y audaz que algunos llegan a pensar que es paralizantc, clitista y tcórica. En el terreno cducativo, indudablemente cl decidir pensar está indisolublemente ligado al decidir hacer y sentir que presuponen no solamente la construcción de la acción colectiva sino también la recuperación de la temura y de los proccsos de humanización en las más amplias accpciones del término.

En nuestro siglo y frente a esta sociedad que se niega a reconocer la diversidad y en aras de reconstruir el sentido, pertinencia y pertenencia de la educación se pueden comenzar a esbozar algunos principios orientadores de una nueva docencia desde la Pedagogía Crítica que recupcrarán la idea de la cducación 
como proceso de apropiación de la historia y de la cultura, a saber: educar para la incertidumbre, la complejidad y la imprevisibilidad; educar para gozar de la vida; educar para la significación, la coherencia y la integralidad; educar para la expresión y, finalmente, educar para la convivencia y la tolerancia.

\section{Referencias}

Alvarado, R., Cubcro, F., Frías, C., G6mez, K., Mata, A., Monge, M., Pereira, T. (2000). Modulo Educación de Calidad. Serie Rendimiento Escolar: Proyecto Ampliación de la Capacitación del Sistema Educativo Costarricense en Comunidades Afectadas por Migración Nicaragüense relacionadas con el Huracán Mitch. San José, Costa Rica: Agencia para el Desarrollo Interamericano del Gobierno de los Estados Unidos. Asociación Demográfica Costarricense. Ministerio de Educación Pública. Organización Interamericana para las Migraciones. Universidad de Costa Rica.

Ayuste, A., Flecha, R., López, F., y Lleras, J. (1998). Planteamientos de la Pedagogía Crítica. Comunicar y Transformar. Barcelona: Grab.

Florez, R. (1999). Evaluación peclagógica y cognición. Bogotá: Mc Graw Hill.

Rama, G. (1984). Educación, participación y estilos de desarrollo en América Latina. Buenos Aires: Kapelusz-CEPAL.

Sales, A. y García, R. (1997). Programas de educación intercultural. Bilbao: Desclée de Brouwer, S.A.

Sandoval, C. (2003). Otros amenazantes: los nicaragüenses y la formación de identidades nacionales en Costa Rica. San José: Editorial de la Universidad de Costa Rica. 